\title{
COMMUNICABLE DISEASES REPORT, NSW: SEPTEMBER 2002
}

\section{TRENDS}

Notifications of communicable diseases through to the end of July (Figure 2 and Table 1) followed typical midwinter patterns in NSW. Notifications of arbovirus infections and cryptosporidiosis were relatively few, but notifications of invasive pneumococcal disease, meningococcal disease, and influenza, were relatively frequent.

\section{ENHANCED INFLUENZA SURVEILLANCE}

To 3 August 2002, data from sentinel influenza surveillance sites in NSW showed increasing influenza A activity but decreasing influenza B activity in NSW (Figure 1).

For the week ending 3 August, 52 sentinel general practitioners in NSW reported diagnosing 123 patients with influenza-like illnesses [ILIs] among 5,469 total consultations (or 22.5 per 1,000 consultations). This is lower than the number of ILIs reported in the previous week. In the same week, six sentinel laboratories reported that 100 respiratory samples tested positive for influenza $A$ and 10 for influenza $B$ by either direct immunofluorescence or culture (a rate for influenza A, 16.9 positive per 100 samples; and for influenza B, 1.7 positive per 100 samples). These data represent an increase in the rate for influenza A but a decrease in the rate for influenza B) compared with the previous week.

\section{SYPHILIS REPORTS}

There have been several notifications of new cases of syphilis diagnosed in inner Sydney. Below are summaries of these cases from the staff of the Central Sydney and South Eastern Sydney Public Health Units.

The South Eastern Sydney Public Health Unit (SESPHU) began enhanced surveillance for syphilis in 2001. In 2001, 203 syphilis notifications were received by the SESPHU. Of these, 19 cases were classified as having early syphilis (nine with the symptoms of primary syphilis [with chancre], four with the symptoms of secondary syphilis [with rash], and six with latent [asymptomatic] syphilis of $<1$ year's duration). For 2002 to date, 119 syphilis notifications have been received (11 primary, four secondary, and four $<1$ year's duration).

The Sydney Sexual Health Centre, a major public clinic in the SESPHU, reports that early syphilis cases during 2001 were predominantly acquired overseas in countries with epidemics of syphilis among men who have sex with men. Some cases were also acquired locally, through sexual contact with visitors from these countries. Anecdotal reports from the Centre suggest that the number

\section{FIGURE 1}

REPORTS OF INFLUENZA DIAGNOSES BY SENTINEL LABORATORIES, NSW 1999-2002

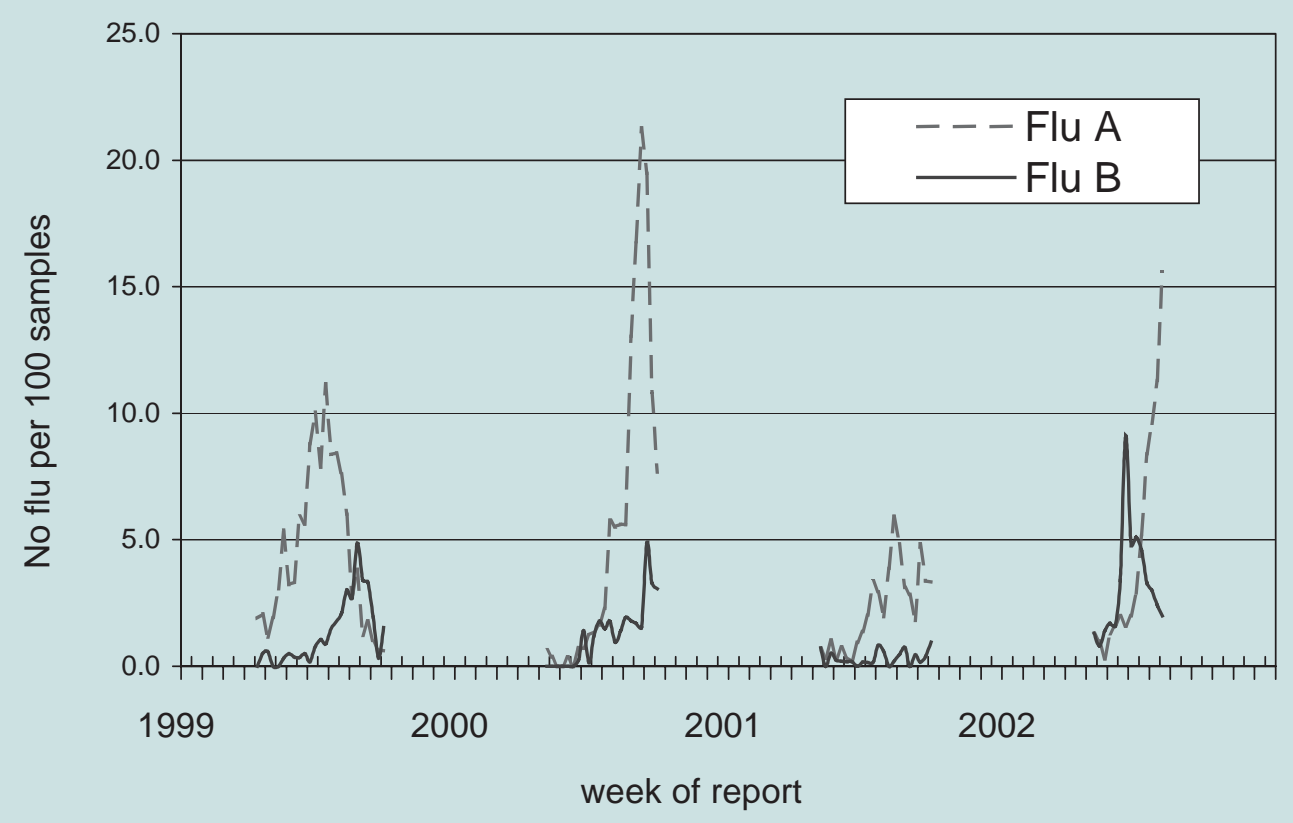

Source: Communicable Diseases Branch, NSW Department of Health. 
of locally-acquired cases in men has increased in 2002.

The Central Sydney Public Health Unit (CSPHU) began enhanced surveillance for syphilis in April 1999. CSPHU gathers information from the doctors of patients who have a positive serological result for syphilis. Data collected includes basic demographic information about the patient and information about the stage of disease.

The number of early syphilis cases has steadily increased from six in 1999 to 14 for the first seven months of 2002. Since March 2002 enhanced surveillance has identified six cases of primary or secondary syphilis, including four cases with chancre and two cases with rash, spots, and/or lymphadenopathy. The four cases with chancre have been diagnosed since June. All four were men and two of these individuals had acquired syphilis overseas. There was no link identified among these cases. Sexual Health Services in Central Sydney have since notified CSPHU of an additional four cases of primary syphilis in men who have sex with men.

This increase in the number of cases of early syphilis is consistent with trends in Europe, North America, and Britain, where increases in cases and outbreaks-often including men who have sex with men-have been reported since $1997 .{ }^{1}$

The diagnostic classification of syphilis cases requires the interpretation of often-complex serological results and patient histories, leading to the possibility of misclassification of cases. However, it is likely that many of these cases were infectious and these reports may be a harbinger of further cases. Both the SESPHU and the CSPHU will be working closely with the NSW Department of Health, the AIDS Council of NSW, and the Sexually Transmitted Infections in Gay Men's Action Group (STIGMA) to develop prevention strategies.

\section{Reference}

1. Nicoll A, Hamers FH. Are trends in HIV, gonorrhoea, and syphilis worsening in western Europe? BMJ 2002; 324: $1324-1327$.

\section{FOCUS ON ENTERIC DISEASE}

Enteric infections due to viruses, bacteria, parasites, and toxins may be transmitted via food and water and from person-to-person (directly and indirectly). Currently, 10 enteric infections are notifiable in NSW: botulism, cholera, cryptosporidiosis, giardiasis, haemolytic uraemic syndrome, vero-toxigenic E. coli infections, hepatitis A, hepatitis E, listeriosis, and salmonellosis (including typhoid or paratyphoid).

Ms Jennie Musto has joined the Communicable Diseases Branch as epidemiologist for enteric infections. Future editions of the NSW Public Health Bulletin will include summaries of the surveillance for enteric infections. Readers' comments or suggestions for the content of this section would be appreciated. 


\section{FIGURE 2}

\section{REPORTS OF SELECTED COMMUNICABLE DISEASES, NSW, JANUARY 1997 TO JULY 2002,}

BY MONTH OF ONSET

These are preliminary data: case counts for recent months may increase because of reporting delays. Laboratory-confirmed cases, except for measles, meningococcal disease

\begin{tabular}{|rc|}
\hline \multicolumn{3}{|c|}{ NSW population } \\
Male & $50 \%$ \\
$<5$ & $7 \%$ \\
$5-24$ & $28 \%$ \\
$25-64$ & $52 \%$ \\
$65+$ & $13 \%$ \\
Rural $^{*}$ & $42 \%$ \\
\hline
\end{tabular}

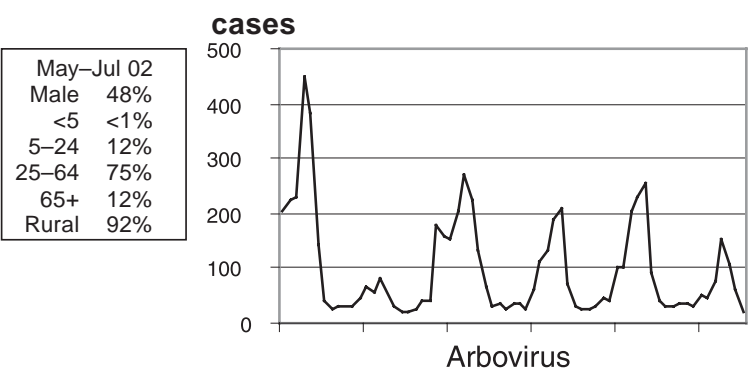

\section{cases}

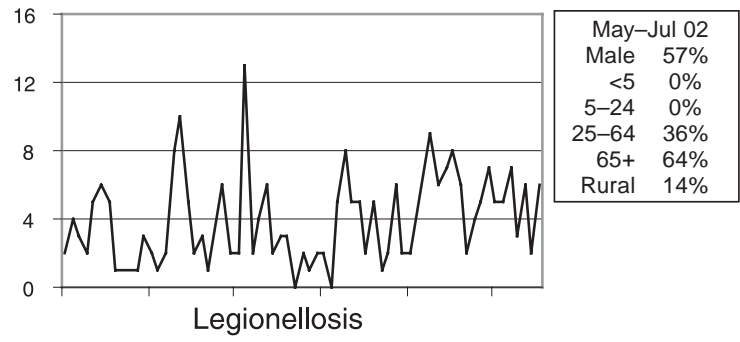

\begin{tabular}{|rr|}
\hline \multicolumn{2}{|c|}{ May-Jul 02 } \\
Male & $54 \%$ \\
$<5$ & $37 \%$ \\
$5-24$ & $35 \%$ \\
$25-64$ & $26 \%$ \\
$65+$ & $2 \%$ \\
Rural & $52 \%$ \\
\hline
\end{tabular}
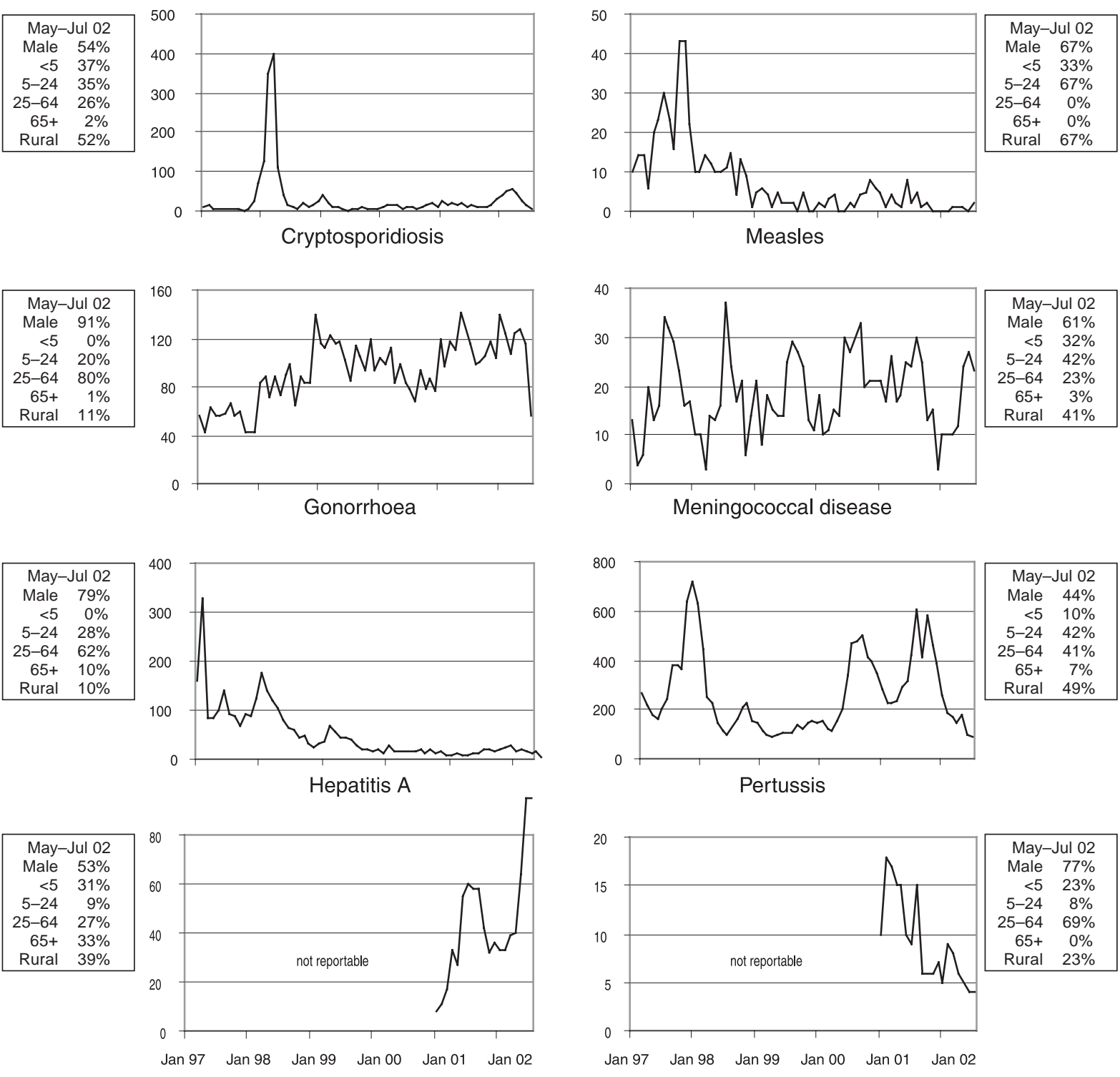

Invasive Pneumococcal disease

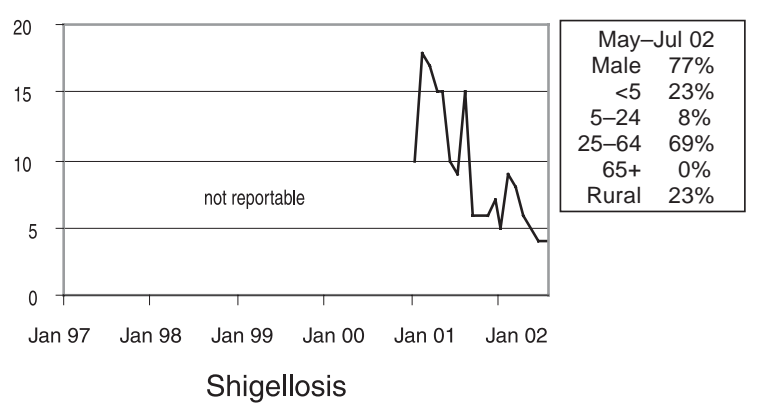




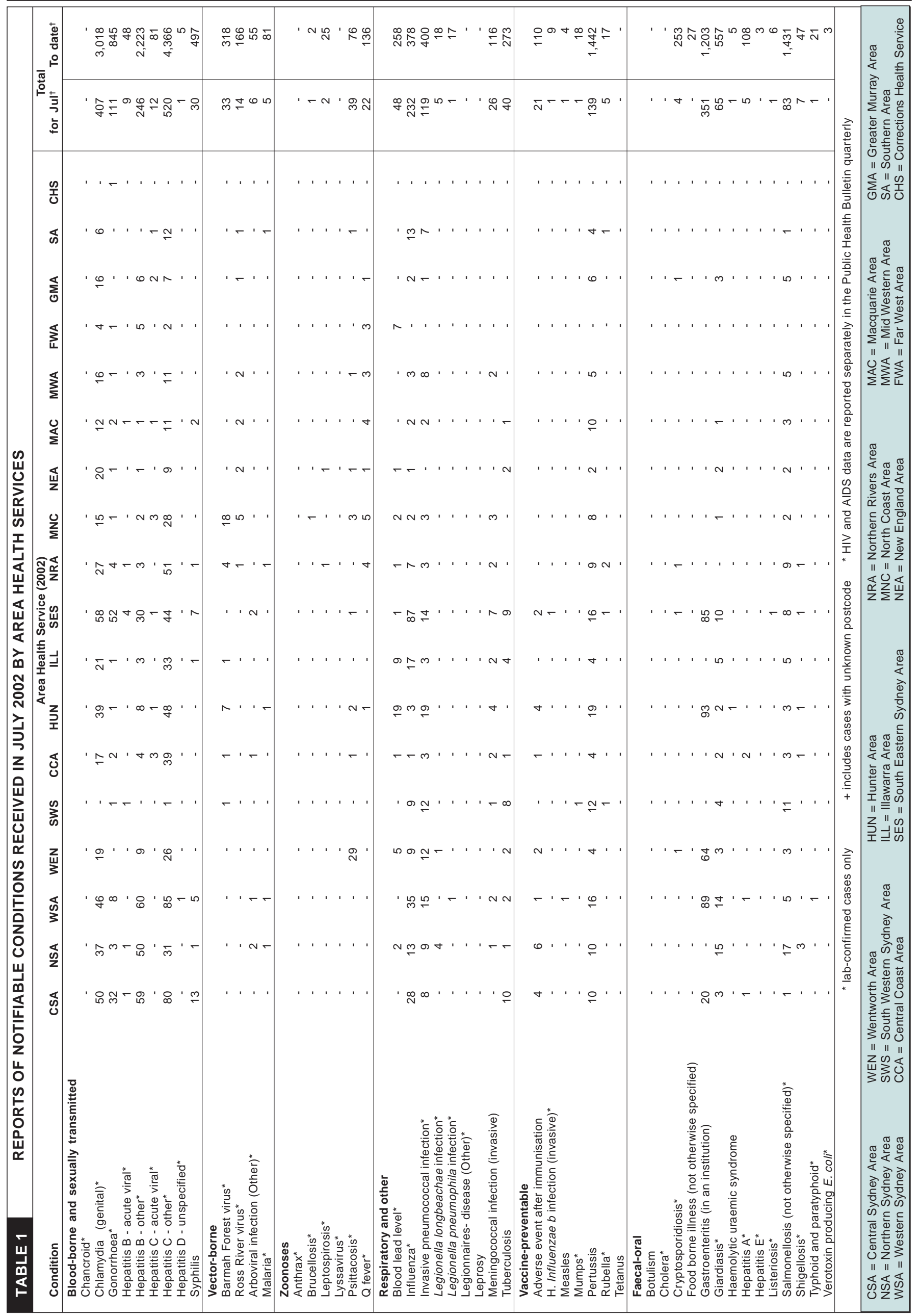

